\title{
HIGHLIGHTS
}

BONE

\section{Hip fracture risk and cardiovascular disease}

A diagnosis of cardiovascular disease is strongly associated with an increased risk of hip fracture, and this association seems to be mediated by genetic factors, say researchers from Sweden.

\section{4 ...all major cardiovascular} diseases are independently associated with a substantial future increased risk of hip fracture... 77

A cohort of 15,968 Swedish twin pairs born between 1914 and 1944 and alive in 1972 was used to analyze the association between different cardiovascular diseases (heart failure, stroke, ischemic heart disease, peripheral atherosclerosis and hypertension) and future risk of hip fracture. Information on fractures, diagnosis of cardiovascular disease and comorbidities were determined in study participants aged $\geq 50$ years, who were followed up until the date of a first hip fracture, death or the end of the study period (December 2005).

The crude absolute rate of hip fractures was 12.6 per 1,000 person-years after a diagnosis of heart failure (hazard ratio 4.40, 95\% CI 3.43-5.63) compared with 1.2 per 1,000 person-years for individuals without cardiovascular diseases.

In a secondary analysis, the group of Karl Michaëlsson (Uppsala University, Sweden) investigated whether healthy twin siblings of twins previously diagnosed with cardiovascular disease were also at an increased risk of future fracture. An increased risk of hip fracture in the twin without cardiovascular disease, especially in identical twins, would indicate that the association is probably owing to genetic variation rather than lifestyle.

"We could show that all major cardiovascular diseases are independently associated with a substantial future increased risk of hip fracture, the most devastating type of all osteoporotic fractures," explains Michaëlsson.

"We were also able to display, by use of the twin design, that most of the increased risk of hip fracture after a diagnosis of heart failure and stroke is explained by genes (or early environmental sharing), not lifestyle."

Ongoing studies by Michaëlsson and colleagues will attempt to identify which specific genes or factors contribute to the association between cardiovascular disease and increased hip fracture risk. "In addition, we are trying to evaluate the association between more proximal measures of cardiovascular function (for example, endothelial function) and BMD and fractures," concludes Michaëlsson.

\section{Linda Koch}

Original article Sennerby, U. et al. Cardiovascular diseases and risk of hip fracture. JAMA 302, 1666-1673 (2009) 